\title{
Strong Convergence to Common Fixed Points of a Countable Family of Asymptotically Strictly Quasi- $\phi$-Pseudocontractions
}

\author{
Wei-Qi Deng \\ College of Statistics and Mathematics, Yunnan University of Finance and Economics, Kunming, Yunnan 650221, China \\ Correspondence should be addressed to Wei-Qi Deng; dwq1273@126.com
}

Received 8 July 2013; Accepted 11 September 2013

Academic Editor: Sotiris Ntouyas

Copyright (C) 2013 Wei-Qi Deng. This is an open access article distributed under the Creative Commons Attribution License, which permits unrestricted use, distribution, and reproduction in any medium, provided the original work is properly cited.

Based on an original idea, namely, a specific way of choosing the indexes of the involved mappings, we propose a new hybrid shrinking iteration scheme for approximating some common fixed points of a countable family of asymptotically strictly quasi- $\phi$-pseudocontractions and obtain a strong convergence theorem in the framework of Banach space. Our result extends other authors, related results existing in the current literature. As application, an iterative solution to a system of equilibrium problems is provided.

\section{Introduction}

Fixed point theory as an important branch of nonlinear analysis theory has been applied in many disciplines. From the viewpoint of applications in real world, it is not only to show the existence of fixed points of nonlinear mappings but also to construct iterative algorithms to approximate their fixed points, which explains the popularity of iteration methods for finding the fixed points of various nonlinear mappings. For instance, iteration approximation of common fixed points of asymptotically nonexpansive mappings, an important class of nonlinear mappings introduced by Goebel and Kirk [1], has been studied by many authors (see, e.g., [2-7]).

In this paper, we discuss the approximation of common fixed points of a family of asymptotically strictly quasi- $\phi$ pseudocontractions.

Throughout this paper we assume that $E$ is a real Banach space with dual $E^{*}, C$ is a nonempty closed convex subset of $E$, and $J: E \rightarrow 2^{E^{*}}$ is the normalized duality mapping defined by

$$
J x=\left\{f \in E^{*}:\langle x, f\rangle=\|x\|^{2}=\|f\|^{2}\right\}, \quad \forall x \in E .
$$

In the sequel, we use $F(T)$ to denote the set of fixed points of a mapping $T$.
Definition 1 (see [8]). A mapping $T: C \rightarrow C$ is said to be an asymptotically strictly quasi- $\phi$-pseudocontraction, if $F(T) \neq \emptyset$, and there exists a nonnegative real sequence $\left\{\mu_{n}\right\}$ with $\mu_{n} \rightarrow 0$ (as $n \rightarrow \infty$ ) and a constant $\kappa \in[0,1)$ such that

$$
\begin{array}{r}
\phi\left(p, T^{n} x\right) \leq\left(1+\mu_{n}\right) \phi(p, x)+\kappa \phi\left(x, T^{n} x\right), \\
\forall n \geq 1, x \in C, p \in F(T),
\end{array}
$$

where $\phi: E \times E \rightarrow \mathbb{R}^{+}$denotes the Lyapunov functional defined by

$$
\phi(x, y)=\|x\|^{2}-2\langle x, J y\rangle+\|y\|^{2}, \quad \forall x, y \in E,
$$

where $\langle\cdot, \cdot\rangle$ stands for the duality product. It is obvious from the definition of $\phi$ that

$$
\begin{array}{r}
\phi(x, y)=\phi(x, z)+\phi(z, y)+2\langle x-z, J z-J y\rangle, \\
\forall x, y, z \in E, \\
(\|x\|-\|y\|)^{2} \leq \phi(x, y) \leq(\|x\|+\|y\|)^{2}, \quad \forall x, y \in E .
\end{array}
$$

Example 2. Let $C$ be a unit ball in a real Hilbert space $l^{2}$, and let $T: C \rightarrow C$ be a mapping defined by

$$
T\left(x_{1}, x_{2}, \ldots\right)=\left(0, x_{1}^{2}, a_{2} x_{2}, a_{3} x_{3}, \ldots\right),
$$


where $\left\{a_{i}\right\}$ is a sequence in $(0,1)$ satisfying $\prod_{i=2}^{\infty} a_{i}=1 / 2$. It is shown in [8] that

$$
\begin{array}{r}
\phi\left(p, T^{n} y\right) \leq\left(1+\mu_{n}\right) \phi(p, y), \\
\forall n \geq 1, y \in C, p \in F(T),
\end{array}
$$

where $\phi(x, y)=\|x-y\|^{2}, \mu_{n}=\left(2 \prod_{i=2}^{n} a_{i}\right)^{2}-1$ for all $n \geq 1$. This shows that the mapping $T$ defined above is an asymptotically strictly quasi- $\phi$-pseudocontraction.

Definition 3 (see [9]). A mapping $T: C \rightarrow C$ is said to be asymptotically regular on $C$ if, for any bounded subset $K$ of $C$,

$$
\lim _{n \rightarrow \infty} \sup _{x \in K}\left\{\left\|T^{n+1} x-T^{n} x\right\|\right\}=0 .
$$

In 2012, Zhang [9] used the following hybrid projection algorithm for a single asymptotically strictly quasi- $\phi$-pseudocontraction to obtain strong convergence under a limit condition only in the framework of reflexive, strictly convex, and smooth Banach spaces such that both $E$ and $E^{*}$ have the Kadec-Klee property

$$
\begin{gathered}
x_{0} \in E \text { chosen arbitrarily; } C_{1}=C, \\
x_{1}=\Pi_{C_{1}} x_{0}, \\
C_{n+1}=\left\{u \in C_{n}: \phi\left(x_{n}, T^{n} x_{n}\right)\right. \\
\left.\leq \frac{2}{1-\kappa}\left\langle x_{n}-u, J x_{n}-J T^{n} x_{n}\right\rangle+\mu_{n} \frac{M_{n}}{1-\kappa}\right\}, \\
x_{n+1}=\prod_{C_{n+1}} x_{0}, \quad \forall n \geq 1,
\end{gathered}
$$

where $M_{n}=\sup \left\{\phi\left(p, x_{n}\right): p \in F(T)\right\}$ and $\Pi_{C_{n+1}}$ is the generalized projection (see (10)) of $E$ onto $C_{n+1}$. Their results improved the corresponding results of Zhou and Gao [10] and Qin et al. [8].

Inspired and motivated by the studies mentioned above, we introduce an up-to-date iteration scheme for approximating common fixed points of a countable family of asymptotically strictly quasi- $\phi$-pseudocontractions and obtain a strong convergence theorem. The result extends the corresponding one for one map in [9].

\section{Preliminaries}

Following Alber [11], the generalized projection $\Pi_{C}: E \rightarrow C$ is defined by

$$
\Pi_{C}=\arg \inf _{y \in C} \phi(y, x), \quad \forall x \in E
$$

Lemma 4 (see [11]). Let E be a smooth, strictly convex, and reflexive Banach space and $C$ a nonempty closed convex subset of $E$. Then the following conclusions hold:

(1) $\phi\left(x, \Pi_{C} y\right)+\phi\left(\Pi_{C} y, y\right) \leq \phi(x, y)$ for all $x \in C$ and $y \in E$;
(2) if $x \in E$ and $z \in C$, then $z=\Pi_{C} x \Leftrightarrow\langle z-y, J x-J z\rangle \geq$ 0 , for all $y \in C$;

(3) for $x, y \in E, \phi(x, y)=0$ if and only if $x=y$.

Remark 5. The following basic properties for a Banach space $E$ can be found in [12]:

(i) if $E$ is uniformly smooth, then $J$ is uniformly continuous on each bounded subset of $E$;

(ii) if $E$ is reflexive and strictly convex, then $J^{-1}$ is normweak-continuous;

(iii) if $E$ is a smooth, strictly convex, and reflexive Banach space, then the normalized duality mapping $J: E \rightarrow$ $2^{E^{*}}$ is single valued, one-to-one, and onto;

(iv) a Banach space $E$ is uniformly smooth if and only if $E^{*}$ is uniformly convex;

(v) each uniformly convex Banach space $E$ has the KadecKlee property; that is, for any sequence $\left\{x_{n}\right\} \subset E$, if $x_{n} \rightarrow x \in E$ and $\left\|x_{n}\right\| \rightarrow\|x\|$, then $x_{n} \rightarrow x$ as $n \rightarrow \infty$

Lemma 6 (see [13]). Let $E$ be a strictly convex and smooth Banach space, let $C$ be a nonempty and closed convex subset of $E$, and let $T$ be a quasi- $\phi$-nonexpansive mapping from $C$ into $E$. Then $F(T)$ is closed and convex.

Lemma 7 (see [14]). The unique solutions to the positive integer equation

$$
n=i+\frac{(m-1) m}{2}, \quad m \geq i, n=1,2,3, \ldots
$$

are

$$
\begin{gathered}
i=n-\frac{(m-1) m}{2}, \\
m=-\left[\frac{1}{2}-\sqrt{2 n+\frac{1}{4}}\right], \quad n=1,2,3, \ldots,
\end{gathered}
$$

where $[x]$ denotes the maximal integer that is not larger than $x$.

\section{Main Results}

Recall that an operator $T$ in a Banach space is closed if $x_{n} \rightarrow x$ and $T x_{n} \rightarrow y$ as $n \rightarrow \infty$; then $T x=y$.

Theorem 8. Let $E$ be a reflexive, strictly convex, and smooth Banach space such that both $E$ and $E^{*}$ have Kadec-Klee property, let $C$ be a nonempty closed and convex subset of $E$, and $\left\{T_{i}\right\}: C \rightarrow C$ be a countable family of closed and asymptotically strictly quasi- $\phi$-pseudocontractions with a nonnegative real 
sequence $\left\{\mu_{n}^{(i)}\right\}$ satisfying $\mu_{n}^{(i)} \rightarrow 0($ as $n \rightarrow \infty$ for each $i \geq 1)$ and a sequence $\left\{\kappa_{i}\right\} \subset[0,1)$. Let $\left\{x_{n}\right\}$ be the sequence generated by

$$
\begin{gathered}
x_{1} \in E ; \quad C_{1}=C, \\
C_{n+1}=\left\{z \in C_{n}: \phi\left(x_{n}, T_{i_{n}}^{m_{n}} x_{n}\right)\right. \\
\left.\leq \frac{2}{1-\kappa_{i_{n}}}\left\langle x_{n}-z, J x_{n}-J T_{i_{n}}^{m_{n}} x_{n}\right\rangle+\mu_{m_{n}}^{\left(i_{n}\right)} \frac{v_{n}}{1-\kappa_{i_{n}}}\right\}, \\
x_{n+1}=\prod_{C_{n+1}} x_{1}, \quad \forall n \geq 1,
\end{gathered}
$$

where $\nu_{n}:=\sup _{p \in F} \phi\left(p, x_{n}\right) ; \Pi_{C_{n+1}}$ is the generalized projection of $E$ onto $C_{n+1} ; i_{n}$ and $m_{n}$ are the solutions to the positive integer equation $n=i+((m-1) m / 2)(m \geq i, n=1,2, \ldots)$; that is, for each $n \geq 1$, there exist unique $i_{n}$ and $m_{n}$ such that

$$
\begin{gathered}
i_{1}=1, \quad i_{2}=1, \quad i_{3}=2, \quad i_{4}=1, \quad i_{5}=2, \\
i_{6}=3, \quad i_{7}=1, \quad i_{8}=2, \ldots ; \\
m_{1}=1, \quad m_{2}=2, \quad m_{3}=2, \quad m_{4}=3, \\
m_{5}=3, \quad m_{6}=3, \quad m_{7}=4, \quad m_{8}=4, \ldots
\end{gathered}
$$

If $\left\{T_{i}\right\}$ are asymptotically regular on $C$ and $F:=\cap_{i=1}^{\infty} F\left(T_{i}\right)$ is nonempty and bounded, then $\left\{x_{n}\right\}$ converges strongly to $\Pi_{F} x_{1}$.

Proof. We divide the proof into several steps.

(i) Both $F$ and $C_{n}$ (for all $n \geq 1$ ) are closed and convex subsets in $C$.

For each $i \geq 1$, the closedness of $F\left(T_{i}\right)$ follows from that of $T_{i}$, and hence $F$ is closed. In addition, using the same argument as presented in the proof of Theorem 2.1 in [9], we have that each $F_{i}$ is convex and so is $F$.

Next, we show that $C_{n}$ is closed and convex for all $n \geq 1$. With $C_{1}(=C)$ being closed and convex, we may assume that $C_{n}$ is closed and convex for some $n \geq 2$. In view of the definition of $C_{n+1}$ we have that

$$
C_{n+1}=\{z \in C: \varphi(z) \leq a\} \cap C_{n}
$$

where $\varphi(z)=\left(2 / \kappa_{i_{n}}-1\right)\left\langle x_{n}-z, J x_{n}-J T_{i_{n}}^{m_{n}} x_{n}\right\rangle$ and $a=$ $\mu_{m_{n}}^{\left(i_{n}\right)}\left(\nu_{n} / 1-\kappa_{i_{n}}\right)-\phi\left(x_{n}, T_{i_{n}}^{m_{n}} x_{n}\right)$. This shows that $C_{n+1}$ is closed and convex.

(ii) $F$ is a subset of $\cap_{n=1}^{\infty} C_{n}$.

It is obvious that $F \subset C_{1}$. Suppose that $F \subset C_{n}$ for some $n \geq 2$. It follows from (4) that

$$
\begin{aligned}
\phi\left(p, T_{i_{n}}^{m_{n}} x_{n}\right)= & \phi\left(p, x_{n}\right)+\phi\left(x_{n}, T_{i_{n}}^{m_{n}} x_{n}\right) \\
& +2\left\langle p-x_{n}, J x_{n}-J T_{i_{n}}^{m_{n}} x_{n}\right\rangle
\end{aligned}
$$

for any $p \in F \subset C_{n}$. Then, from (16) we have

$$
\begin{aligned}
\phi\left(x_{n}, T_{i_{n}}^{m_{n}} x_{n}\right) \leq & \frac{\mu_{m_{n}}^{\left(i_{n}\right)}}{1-\kappa_{i_{n}}} \phi\left(p, x_{n}\right) \\
& +\frac{2}{1-\kappa_{i_{n}}}\left\langle x_{n}-p, J x_{n}-J T_{i_{n}}^{m_{n}} x_{n}\right\rangle \\
\leq & \mu_{m_{n}}^{\left(i_{n}\right)} \frac{\nu_{n}}{1-\kappa_{i_{n}}} \phi\left(p, x_{n}\right) \\
& +\frac{2}{1-\kappa_{i_{n}}}\left\langle x_{n}-p, J x_{n}-J T_{i_{n}}^{m_{n}} x_{n}\right\rangle .
\end{aligned}
$$

This implies that $p \in C_{n+1}$, and so $F \subset C_{n+1}$.

(iii) Consider $x_{n} \rightarrow x^{*} \in C$ as $n \rightarrow \infty$.

In fact, since $x_{n}=\Pi_{C_{n}} x_{1}$, from Lemma 4 (2) we have $\left\langle x_{n}-y, J x_{1}-J x_{n}\right\rangle \geq 0$, for all $y \in C_{n}$. Again since $F \subset$ $\cap_{n=1}^{\infty} C_{n}$, we have $\left\langle x_{n}-p, J x_{1}-J x_{n}\right\rangle \geq 0$, for all $p \in F$. It follows from Lemma 4 (1) that for each $p \in F$ and for each $n \geq 1$,

$$
\phi\left(x_{n}, x_{1}\right)=\phi\left(\Pi_{C_{n}} x_{1}, x_{1}\right) \leq \phi\left(p, x_{1}\right)-\phi\left(p, x_{n}\right) \leq \phi\left(p, x_{1}\right),
$$

which implies that $\left\{\phi\left(x_{n}, x_{1}\right)\right\}$ is bounded and hence so is $\left\{x_{n}\right\}$. Since for all $n \geq 1, x_{n}=\Pi_{C_{n}} x_{1}$ and $x_{n+1}=\Pi_{C_{n+1}} x_{1} \in$ $C_{n+1} \subset C_{n}$, we have $\phi\left(x_{n}, x_{1}\right) \leq \phi\left(x_{n+1}, x_{1}\right)$. This implies that $\left\{\phi\left(x_{n}, x_{1}\right)\right\}$ is nondecreasing; hence the limit

$$
\lim _{n \rightarrow \infty} \phi\left(x_{n}, x_{1}\right) \text { exists. }
$$

Since $E$ is reflexive, there exists a subsequence $\left\{x_{n_{i}}\right\}$ of $\left\{x_{n}\right\}$ such that $x_{n_{i}} \rightarrow x^{*} \in C$ as $i \rightarrow \infty$. Since $C_{n}$ is closed and convex and $C_{n+1} \subset C_{n}$, this implies that $C_{n}$ is weakly closed and $x^{*} \in C_{n}$ for each $n \geq 1$. In view of $x_{n_{i}}=\prod_{C_{n_{i}}} x_{1}$, we have

$$
\phi\left(x_{n_{i}}, x_{1}\right) \leq \phi\left(x^{*}, x_{1}\right), \quad \forall i \geq 1 .
$$

Since the norm $\|\cdot\|$ is weakly lower semicontinuous, we have

$$
\begin{aligned}
\liminf _{i \rightarrow \infty} \phi\left(x_{n_{i}}, x_{1}\right) & =\liminf _{i \rightarrow \infty}\left(\left\|x_{n_{i}}\right\|^{2}-2\left\langle x_{n_{i}}, J x_{1}\right\rangle+\left\|x_{1}\right\|^{2}\right) \\
& \geq\left\|x^{*}\right\|^{2}-2\left\langle x^{*}, J x_{1}\right\rangle+\left\|x_{1}\right\|^{2} \\
& =\phi\left(x^{*}, x_{1}\right),
\end{aligned}
$$

and so

$$
\begin{aligned}
\phi\left(x^{*}, x_{1}\right) & \leq \liminf _{i \rightarrow \infty} \phi\left(x_{n_{i}}, x_{1}\right) \\
& \leq \limsup _{i \rightarrow \infty} \phi\left(x_{n_{i}}, x_{1}\right) \leq \phi\left(x^{*}, x_{1}\right) .
\end{aligned}
$$

This implies that $\lim _{i \rightarrow \infty} \phi\left(x_{n_{i}}, x_{1}\right)=\phi\left(x^{*}, x_{1}\right)$, and so $\left\|x_{n_{i}}\right\| \rightarrow\left\|x^{*}\right\|$ as $i \rightarrow \infty$. Since $x_{n_{i}} \rightarrow x^{*}$, by virtue of KadecKlee property of $E$, we obtain that

$$
\lim _{i \rightarrow \infty} x_{n_{i}}=x^{*} .
$$


Since $\left\{\phi\left(x_{n}, x_{1}\right)\right\}$ is convergent, this, together with $\lim _{i \rightarrow \infty} \phi\left(x_{n_{i}}, x_{1}\right)=\phi\left(x^{*}, x_{1}\right)$, shows that $\lim _{n \rightarrow \infty} \phi\left(x_{n}, x_{1}\right)$ $=\phi\left(x^{*}, x_{1}\right)$. If there exists some subsequence $\left\{x_{n_{j}}\right\}$ of $\left\{x_{n}\right\}$ such that $x_{n_{j}} \rightarrow y$ as $j \rightarrow \infty$, then from Lemma 4 (1) we have that

$$
\begin{aligned}
\phi\left(x^{*}, y\right) & =\lim _{i, j \rightarrow \infty} \phi\left(x_{n_{i}}, x_{n_{j}}\right)=\lim _{i, j \rightarrow \infty} \phi\left(x_{n_{i}}, \Pi_{C_{n_{j}}} x_{1}\right) \\
& \leq \lim _{i, j \rightarrow \infty}\left(\phi\left(x_{n_{i}}, x_{1}\right)-\phi\left(\Pi_{C_{n_{j}}} x_{1}, x_{1}\right)\right) \\
& =\lim _{i, j \rightarrow \infty}\left(\phi\left(x_{n_{i}}, x_{1}\right)-\phi\left(x_{n_{j}}, x_{1}\right)\right) \\
& =\phi\left(x^{*}, x_{1}\right)-\phi\left(x^{*}, x_{1}\right)=0 .
\end{aligned}
$$

That is, $x^{*}=y$ and so

$$
\lim _{n \rightarrow \infty} x_{n}=x^{*}
$$

(iv) $x^{*}$ is a member of $F$.

Set $\mathbb{N}_{i}=\{k \in \mathbb{N}: k=i+((m-1) m / 2), m \geq i, m \in \mathbb{N}\}$ for each $i \geq 1$. Note that $T_{i_{k}}^{m_{k}}=T_{i}^{m_{k}}, \kappa_{i_{k}}=\kappa_{i}$, and $\mu_{m_{k}}^{\left(i_{k}\right)}=\mu_{m_{k}}^{(i)}$ whenever $k \in \mathbb{N}_{i}$ for each $i \geq 1$. For example, by Lemma 7 and the definition of $\mathbb{N}_{1}$, we have $\mathbb{N}_{1}=\{1,2,4,7,11,16, \ldots\}$ and $i_{1}=i_{2}=i_{4}=i_{7}=i_{11}=i_{16}=\cdots=1$. By virtue of $x_{n+1}=\Pi_{C_{n+1}} x_{1} \in C_{n+1}$, we have

$$
\begin{aligned}
& \phi\left(x_{k}, T_{i}^{m_{k}} x_{k}\right) \\
& \quad \leq \frac{2}{1-\kappa_{i}}\left\langle x_{k}-x_{k+1}, J x_{k}-J T_{i}^{m_{k}} x_{k}\right\rangle+\mu_{m_{k}}^{(i)} \frac{\nu_{k}}{1-\kappa_{i}}
\end{aligned}
$$

for all $k \in \mathbb{N}_{i}$. Note that $\left\{m_{k}\right\}_{k \in \mathbb{N}_{i}}=\{i, i+1, i+2, \ldots\}$; that is, $m_{k} \uparrow \infty$ as $\mathbb{N}_{i} \ni k \rightarrow \infty$. It then follows from (25) that

$$
\lim _{\mathbb{N}_{i} \ni k \rightarrow \infty} \phi\left(x_{k}, T_{i}^{m_{k}} x_{k}\right)=0, \quad \forall i \geq 1 .
$$

In view of (5), we have

$$
\lim _{\mathbb{N}_{i} \ni k \rightarrow \infty}\left(\left\|x_{k}\right\|-\left\|T_{i}^{m_{k}} x_{k}\right\|\right)=0, \quad \forall i \geq 1 .
$$

This, in view of (25), implies that

$$
\lim _{\mathbb{N}_{i} \ni k \rightarrow \infty}\left\|T_{i}^{m_{k}} x_{k}\right\|=\left\|x^{*}\right\|, \quad \forall i \geq 1
$$

which yields that

$$
\lim _{\mathbb{N}_{i} \ni k \rightarrow \infty}\left\|J T_{i}^{m_{k}} x_{k}\right\|=\left\|J x^{*}\right\|, \quad \forall i \geq 1 .
$$

This shows that $\left\{J T_{i}^{m_{k}} x_{k}\right\}_{k \in \mathbb{N}_{i}}$ is bounded for each $i \geq 1$. Note that both $E$ and $E^{*}$ are reflexive. We may assume that $J T_{i}^{m_{k}} x_{k} \rightarrow f \in E^{*}$ as $\mathbb{N}_{i} \ni k \rightarrow \infty$. In view of the reflexivity of $E$, we know that there exists an element $y \in E$ such that $J y=f$. Then we have

$$
\begin{aligned}
\phi\left(x_{k}, T_{i}^{m_{k}} x_{k}\right) & =\left\|x_{k}\right\|^{2}-2\left\langle x_{k}, J T_{i}^{m_{k}} x_{k}\right\rangle+\left\|T_{i}^{m_{k}} x_{k}\right\|^{2} \\
& =\left\|x_{k}\right\|^{2}-2\left\langle x_{k}, J T_{i}^{m_{k}} x_{k}\right\rangle+\left\|J T_{i}^{m_{k}} x_{k}\right\|^{2} .
\end{aligned}
$$

Taking $\lim \inf _{\mathbb{N}_{i} \ni k \rightarrow \infty}$ on both sides of the above equation yields that

$$
\begin{aligned}
0 & \geq\left\|x^{*}\right\|^{2}-2\left\langle x^{*}, f\right\rangle+\|f\|^{2} \\
& =\left\|x^{*}\right\|^{2}-2\left\langle x^{*}, J y\right\rangle+\|J y\|^{2} \\
& =\left\|x^{*}\right\|^{2}-2\left\langle x^{*}, J y\right\rangle+\|y\|^{2} \\
& =\phi\left(x^{*}, y\right) .
\end{aligned}
$$

That is, $x^{*}=y$, which in turn implies that $f=J x^{*}$. It follows that $J T_{i}^{m_{k}} x_{k} \rightarrow J x^{*} \in E^{*}$ as $\mathbb{N}_{i} \ni k \rightarrow \infty$. In the light of the Kadec-Klee property of $E^{*}$, we obtain from (30) that $\lim _{\mathbb{N}_{i} \ni k \rightarrow \infty} J T_{i}^{m_{k}} x_{k}=J x^{*}$. Since $J^{-1}: E^{*} \rightarrow E$ is norm-weakcontinuous, $T_{i}^{m_{k}} x_{k} \rightarrow x^{*}$ as $\mathbb{N}_{i} \ni k \rightarrow \infty$. This implies, from (29) and the Kadec-Klee property of $E$, that

$$
\lim _{\mathbb{N}_{i} \ni k \rightarrow \infty} T_{i}^{m_{k}} x_{k}=x^{*}, \quad \forall i \geq 1 .
$$

Again note that $\left\{m_{k}\right\}_{k \in \mathbb{N}_{i}}=\{i, i+1, i+2, \ldots\}$; that is, $m_{k+1}-1=$ $m_{k}$ whenever $k \in \mathbb{N}_{i}$ for each $i \geq 1$. We then have

$$
\begin{aligned}
& \left\|T_{i}^{m_{k+1}} x_{k}-x^{*}\right\| \\
& \quad \leq\left\|T_{i}^{m_{k+1}} x_{k}-T_{i}^{m_{k}} x_{k}\right\|+\left\|T_{i}^{m_{k}} x_{k}-x_{k}\right\| \\
& \quad=\left\|T_{i}^{m_{k+1}} x_{k}-T_{i}^{m_{k+1}-1} x_{k}\right\|+\left\|T_{i}^{m_{k}} x_{k}-x_{k}\right\| .
\end{aligned}
$$

It follows from (33) and the asymptotic regularity of each $T_{i}$ that

$$
\lim _{\mathbb{N}_{i} \ni k \rightarrow \infty}\left\|T_{i}^{m_{k+1}} x_{k}-x^{*}\right\|=0, \quad \forall i \geq 1
$$

That is,

$$
\lim _{\mathbb{N}_{i} \ni k \rightarrow \infty} T_{i}\left(T_{i}^{m_{k}} x_{k}\right)=x^{*}, \quad \forall i \geq 1 .
$$

In view of the closedness of $T_{i}$, it follows from (33) that $T_{i} x^{*}=$ $x^{*}$, namely, for each $i \geq 1, x^{*} \in F\left(T_{i}\right)$ and hence $x^{*} \in F$.

(v) Consider $x^{*}=\Pi_{F} x_{1}$, and so $x_{n} \rightarrow \Pi_{F} x_{1}$ as $n \rightarrow \infty$.

Put $u=\Pi_{F} x_{1}$. Since $u \in F \subset C_{n}$ and $x_{n}=\Pi_{C_{n}} x_{1}$, we have $\phi\left(x_{n}, x_{1}\right) \leq \phi\left(u, x_{1}\right)$, for all $n \geq 1$. Then

$$
\phi\left(x^{*}, x_{1}\right)=\lim _{n \rightarrow \infty} \phi\left(x_{n}, x_{1}\right) \leq \phi\left(u, x_{1}\right),
$$

which implies that $x^{*}=u$ since $u=\prod_{F} x_{1}$, and hence $x_{n} \rightarrow$ $x^{*}=\Pi_{F} x_{1}$. This completes the proof.

\section{Applications to a System of Equilibrium Problems}

The kind of equilibrium problems is a unifying model for several problems arising in physics, engineering, science optimization, economics, transportation, network and structural analysis, Nash equilibrium problems in noncooperative games, and others. It has been shown that variational inequalities and mathematical programming problems can be viewed as a special realization of the abstract equilibrium problems. 
Many authors have proposed some useful methods to solve the equilibrium problem (EP) and generalized equilibrium problem (GEP) (see, e.g., [15-19]).

Let $E$ be a smooth, strictly convex, and reflexive Banach space, $C$ a nonempty closed and convex subset of $E$, and $\left\{f_{i}\right\}_{i=1}^{\infty}: C \times C \rightarrow \mathbb{R}$ a countable family of bifunctions satisfying the conditions: for each $i \geq 1$,

$\left(A_{1}\right) f_{i}(x, x)=0$

$\left(A_{2}\right) f_{i}$ is monotone; that is, $f_{i}(x, y)+f_{i}(y, x) \leq 0$;

$\left(A_{3}\right) \lim \sup _{t \downarrow 0} f_{i}(x+t(z-x), y) \leq f_{i}(x, y)$;

$\left(A_{4}\right)$ the mapping $y \mapsto f_{i}(x, y)$ is convex and lower semicontinuous.

A system of equilibrium problems for $\left\{f_{i}\right\}_{i=1}^{\infty}$ is to find an $x^{*} \in$ $C$ such that

$$
f_{i}\left(x^{*}, y\right) \geq 0, \quad \forall y \in C, i \geq 1,
$$

whose set of common solutions is denoted by EP := $\cap_{i=1}^{\infty} \operatorname{EP}\left(f_{i}\right)$, where $\operatorname{EP}\left(f_{i}\right)$ denotes the set of solutions to the equilibrium problem for $f_{i} \quad(i=1,2, \ldots)$. It will be shown in Theorem 9 that such a system of problems can be reduced to approximation of some fixed points of a countable family of nonlinear mappings.

Let $r>0$. Define a countable family of mappings $\left\{T_{i}\right\}_{i=1}^{\infty}$ : $C \rightarrow C$ as follows:

$$
T_{i}(x)=\left\{z \in C: f_{i}(z, y)+\frac{1}{r}\langle y-z, J z-J x\rangle \geq 0, \forall y \in C\right\},
$$

It is shown in [15] that

(1) $\left\{T_{i}\right\}_{i=1}^{\infty}$ is a sequence of single-valued mappings;

(2) $\left\{T_{i}\right\}_{i=1}^{\infty}$ is a sequence of closed quasi- $\phi$-nonexpansive mappings; that is,

$$
\phi\left(p, T_{i} x\right) \leq \phi(p, x), \quad \forall x \in C, p \in F\left(T_{i}\right) ;
$$

(3) $F:=\cap_{i=1}^{\infty} F\left(T_{i}\right)=\mathrm{EP}$.

Now, we have the following result.

Theorem 9. Let $E$ be a reflexive, strictly convex, and smooth Banach space such that both $E$ and $E^{*}$ have Kadec-Klee property, $C$ a nonempty closed and convex subset of $E$, and $\left\{T_{i}\right\}_{i=1}^{\infty}$ : $C \rightarrow C$ be a countable family of mappings defined by (39). Let $\left\{x_{n}\right\}$ be the sequence generated by

$$
\begin{gathered}
x_{1} \in E ; \quad C_{1}=C, \\
C_{n+1}=\left\{z \in C_{n}: \phi\left(x_{n}, T_{i_{n}} x_{n}\right) \leq 2\left\langle x_{n}-z, J x_{n}-J T_{i_{n}} x_{n}\right\rangle\right\}, \\
x_{n+1}=\prod_{C_{n+1}} x_{1}, \quad \forall n \geq 1,
\end{gathered}
$$

where $\Pi_{C_{n+1}}$ is the generalized projection of $E$ onto $C_{n+1}$; $i_{n}$ is the solution to the positive integer equation $n=i+((m-1) m /$ 2) $(m \geq i, n=1,2, \ldots)$. If $F:=\bigcap_{i=1}^{\infty} F\left(T_{i}\right) \neq \emptyset$, then $\left\{x_{n}\right\}$ strongly converges to $\Pi_{F} x_{1}$ which is a common solution of the system of equilibrium problems for $\left\{f_{i}\right\}_{i=1}^{\infty}$.
Proof. Firstly, it follows from Lemma 6 that $F$ is closed and convex. Secondly, $\left\{T_{i}\right\}_{i=1}^{\infty}$ are obviously asymptotically strictly quasi- $\phi$-pseudocontractions with $\mu_{1}^{(i)}=0$ and $\kappa_{i}=0$ for all $i \geq 1$, since they are quasi- $\phi$-nonexpansive. Finally, (13) clearly reduces to (41) with $m_{n}=1$ for all $n \geq 1$. Therefore conclusion can be obtained immediately from Theorem 8 .

\section{Acknowledgments}

The author is very grateful to the referees for their useful suggestions, by which the content of this paper has been improved. This work is supported by the General Project of Scientific Research Foundation of Yunnan University of Finance and Economics (YC2013A02).

\section{References}

[1] K. Goebel and W. A. Kirk, "A fixed point theorem for asymptotically nonexpansive mappings," Proceedings of the American Mathematical Society, vol. 35, pp. 171-174, 1972.

[2] B. Xu and M. A. Noor, "Fixed-points iteration for asymptotically nonexpansive mappings in Banach spaces," Journal of Mathematical Analysis and Applications, vol. 267, no. 2, pp. 444-453, 2002.

[3] Y. J. Cho, H. Zhou, and G. Guo, "Weak and strong convergence theorems for three-step iterations with errors for asymptotically nonexpansive mappings," Computers \& Mathematics with Applications, vol. 47, no. 4-5, pp. 707-717, 2004.

[4] Z. Q. Liu and S. M. Kang, "Weak and strong convergence for fixed points of asymptotically non-expansive mappings," Acta Mathematica Sinica, vol. 20, no. 6, pp. 1009-1018, 2004.

[5] H. Fukhar-Ud-Din and S. H. Khan, "Convergence of two-step iterative scheme with errors for two asymptotically nonexpansive mappings," International Journal of Mathematics and Mathematical Sciences, vol. 2004, no. 37, pp. 1965-1971, 2004.

[6] S. H. Khan and W. Takahashi, "Approximating common fixed points of two asymptotically nonexpansive mappings," Scientiae Mathematicae Japonicae, vol. 53, no. 1, pp. 143-148, 2001.

[7] H. Fukhar-Ud-Din and A. R. Khan, "Approximating common fixed points of asymptotically nonexpansive maps in uniformly convex Banach spaces," Computers \& Mathematics with Applications, vol. 53, no. 9, pp. 1349-1360, 2007.

[8] X. Qin, T. Wang, and S. Y. Cho, "Hybrid projection algorithms for asymptotically strict quasi- $\phi$-pseudocontractions," Abstract and Applied Analysis, vol. 2011, Article ID 142626, 13 pages, 2011.

[9] Q. N. Zhang, "Strong convergence theorems for fixed points of asymptotically strict quasi- $\phi$-pseudocontractions," Fixed Point Theory and Applications, vol. 2012, article 137, 2012.

[10] H. Zhou and X. Gao, "An iterative method of fixed points for closed and quasi-strict pseudo-contractions in Banach spaces," Journal of Applied Mathematics and Computing, vol. 33, no. 1-2, pp. 227-237, 2010.

[11] Y. I. Alber, "Metric and generalized projection operators in Banach spaces: properties and applications," in Theory and Applications of Nonlinear Operators of Accretive and Monotone Type, A. G. Kartosator, Ed., vol. 178 of Lecture Notes in Pure and Applied Mathematics, pp. 15-50, Marcel Dekker, New York, NY, USA, 1996. 
[12] I. Cioranescu, Geometry of Banach Spaces, Duality Mappings and Nonlinear Problems, vol. 62 of Mathematics and Its Applications, Kluwer Academic, Dordrecht, The Netherlands, 1990.

[13] W. Nilsrakoo and S. Saejung, "Strong convergence to common fixed points of countable relatively quasi-nonexpansive mappings," Fixed Point Theory and Applications, vol. 2008, Article ID 312454, 19 pages, 2008.

[14] W. Q. Deng and P. Bai, "An implicit iteration process for common fixed points of two infinite families of asymptotically nonexpansive mappings in Banach spaces," Journal of Applied Mathematics, vol. 2013, Article ID 602582, 6 pages, 2013.

[15] E. Blum and W. Oettli, "From optimization and variational inequalities to equilibrium problems," The Mathematics Student, vol. 63, no. 1-4, pp. 123-145, 1994.

[16] C. Jaiboon and P. Kumam, "A hybrid extragradient viscosity approximation method for solving equilibrium problems and fixed point problems of infinitely many nonexpansive mappings," Fixed Point Theory and Applications, vol. 2009, Article ID 374815, 32 pages, 2009.

[17] Q.-Y. Liu, W.-Y. Zeng, and N.-J. Huang, "An iterative method for generalized equilibrium problems, fixed point problems and variational inequality problems," Fixed Point Theory and Applications, vol. 2009, Article ID 531308, 20 pages, 2009.

[18] J.-W. Peng and J.-C. Yao, "A viscosity approximation scheme for system of equilibrium problems, nonexpansive mappings and monotone mappings," Nonlinear Analysis: Theory, Methods \& Applications, vol. 71, no. 12, pp. 6001-6010, 2009.

[19] H. Fukhar-Ud-Din, A. R. Khan, and M. A. A. Khan, "Mosco convergence results for common fixed point problems and generalized equilibrium problems in Banach spaces," Journal of Applied Mathematics. In press. 


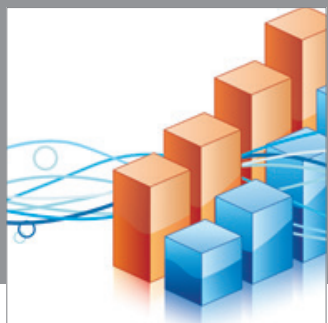

Advances in

Operations Research

mansans

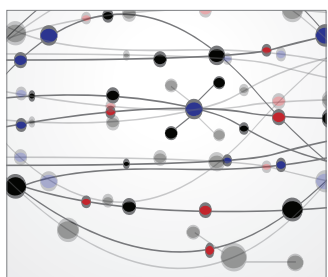

The Scientific World Journal
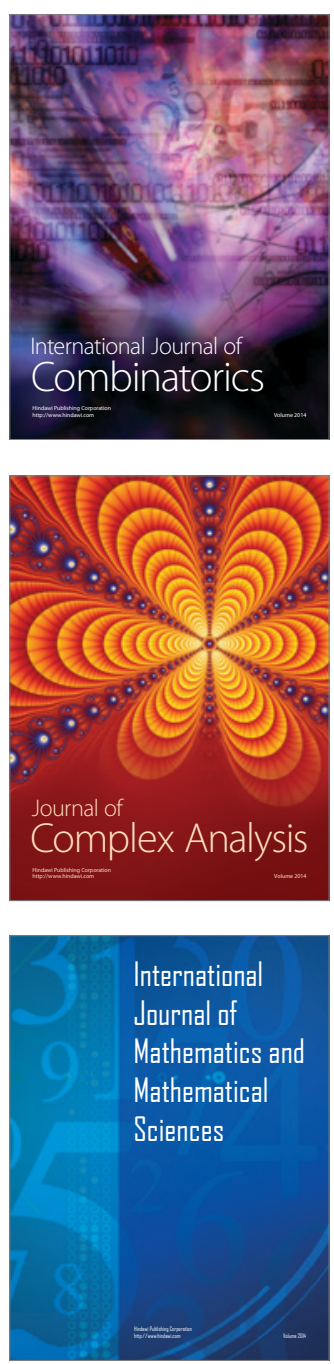
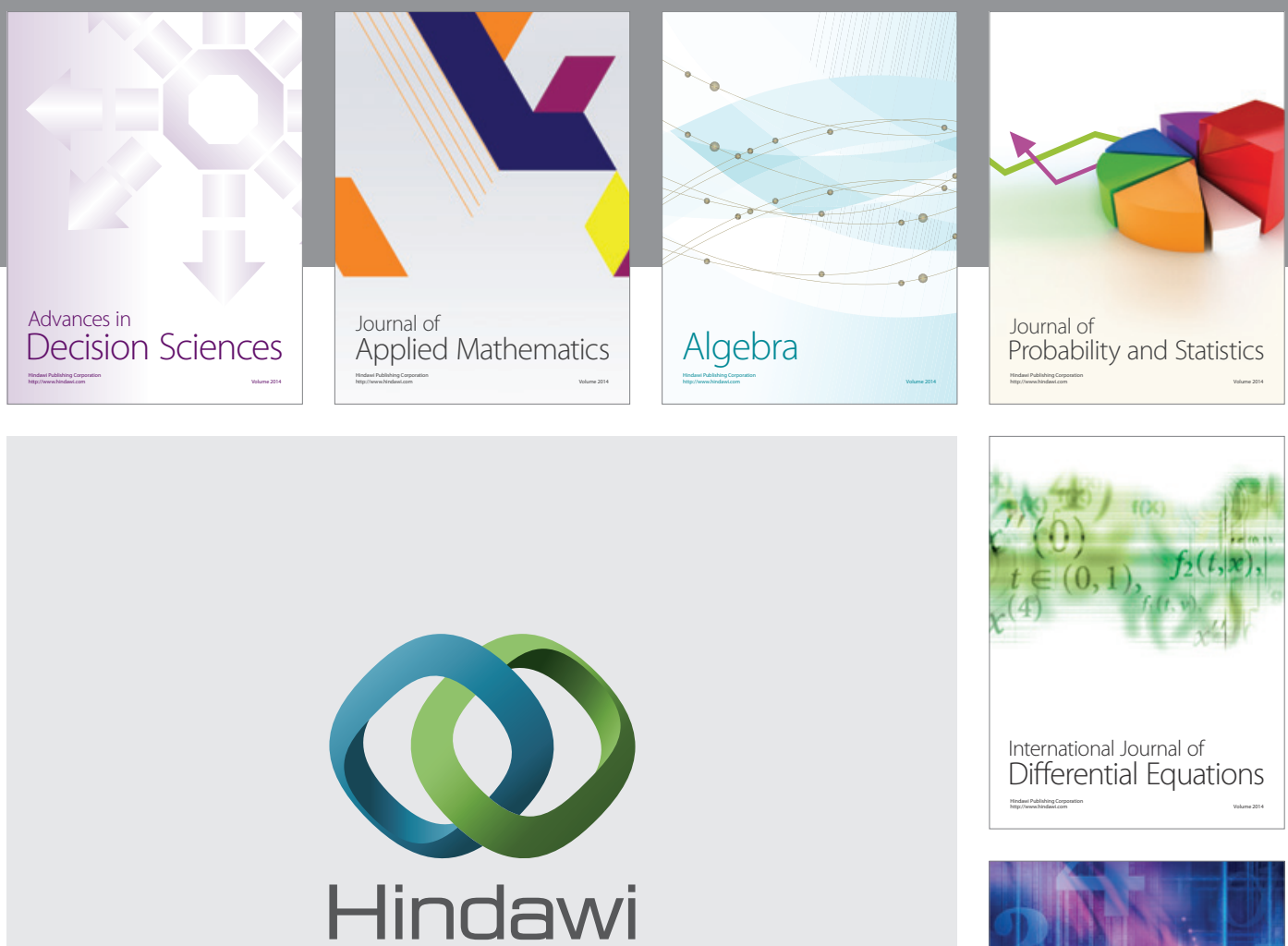

Submit your manuscripts at http://www.hindawi.com
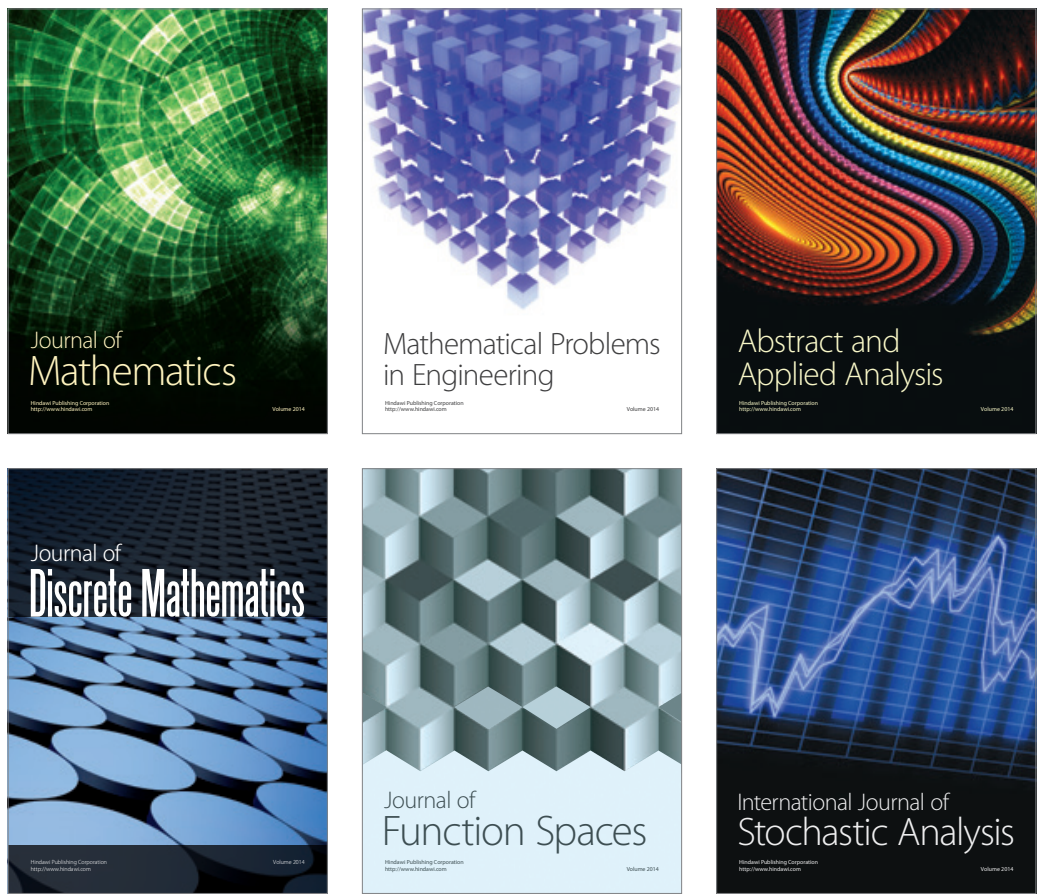

Journal of

Function Spaces

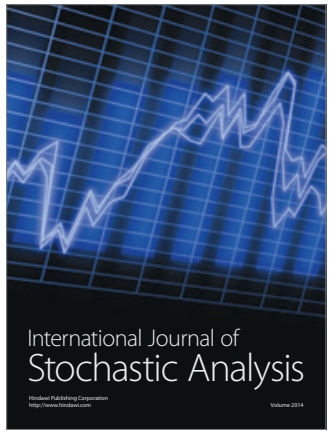

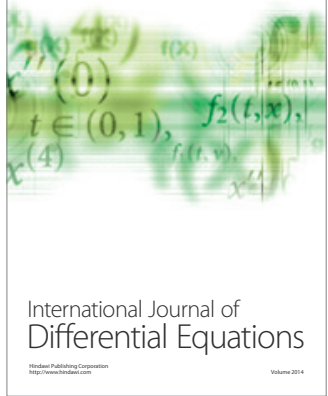
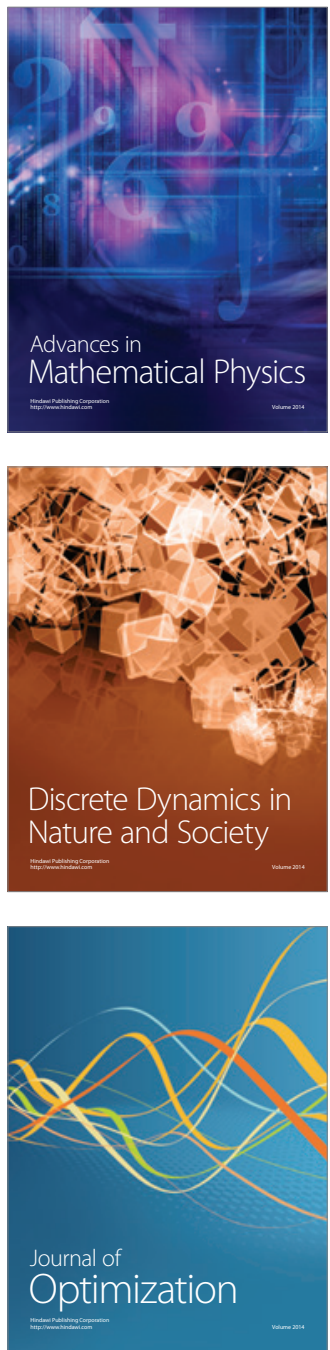